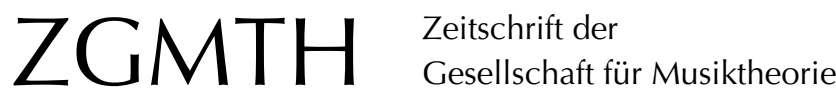

Hust, Christoph (2008): Schenker-Traditionen. Eine Wiener Schule der Musiktheorie und ihre internationale Verbreitung / A Viennese School of Music Theory and Its International Dissemination (= Wiener Veröffentlichungen zur Musikgeschichte 6), hg. von Martin Eybl und Evelyn Fink-Mennel, Wien u.a.: Böhlau 2006. ZGMTH 5/2-3, 411415. https://doi.org/10.31751/296

(C) 2008 Christoph Hust

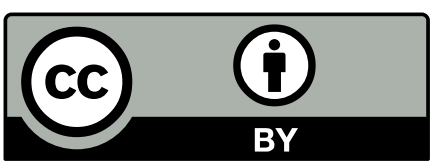

Dieser Text erscheint im Open Access und ist lizenziert unter einer Creative Commons Namensnennung 4.0 International Lizenz.

This is an open access article licensed under a Creative Commons Attribution 4.0 International License.

veröffentlicht / first published: 01/12/2008

zuletzt geändert / last updated: 03/08/2009 


\section{Schenker-Traditionen. Eine Wiener Schule der Musiktheorie und ihre internationale Verbreitung / A Viennese School of Music Theory and Its International Dissemination (= Wiener Veröffentlichungen zur Mu- sikgeschichte 6), hg. von Martin Eybl und Evelyn Fink-Mennel, Wien}

\section{u.a.: Böhlau 2006}

Martin Eybl und Evelyn Fink-Mennel haben bei Böhlau die Vorträge des Wiener Symposiums Schenker-Traditionen aus dem Jahre 2003 im Druck vorgelegt. Der Band wird von einer CD-ROM begleitet, die die Ausstellung Rebell und Visionär. Heinrich Schenker in Wien dokumentiert. Dazu war schon 2003 ein kleiner Katalog gleichen Titels erschienen, der sich inhaltlich mit dem neuen Buch in Teilen überschneidet. Die 16 Beiträge widmen sich der vornehmlich US-amerikanischen Rezeption von Schenkers Schichtenlehre bis zur zweiten Schülergeneration. So disparat diese im Spannungsfeld der zwei einander in herzlicher Abneigung verbundenen Deutungsschulen Salzer und Jonas/Oster verlief, so unterschiedlich sind (thematisch wie auch methodisch) die Artikel angelegt. Martin Eybls konzise Einleitung unternimmt es, den roten Faden zu spannen und diverse Lesarten der schenkerschen Theorie, ihre Anpassung an neue gedankliche Kontexte, als stetige Interpretation und Neudeutung zu verstehen. Dieser Ton ist (entgegen Robert Snarrenbergs kontrovers diskutiertem stheorie-konservativem « "Competing Myths«Artikel von 1994) auch den meisten anderen Texten gemeinsam. Eybl und Fink-Mennel haben das Buch in drei inhaltliche Sektionen gegliedert: "Schenker und Schönberg« - »Proponenten und Schulen « - "Schenker-Lektüre in den USA: Editionen und Übersetzungen«. Bei aller Verschiedenartigkeit schälen sich bei der Lektüre zwei Kernprobleme heraus: der Umgang mit dem weltanschaulichen Kontext von Schenkers Theorie und die Frage ihrer Übertragbarkeit auf prä- und vor allem posttonale Musik.

\section{I. »Schenker und Schönberg»}

Nicht weniger als sechs Autoren beleuchten das schwierige Verhältnis von Schenkers und Schönbergs Konzeptionen der Musiktheorie, und auf ihre Beiträge möchte ich hier etwas ausführlicher eingehen. Zunächst untersucht Bryan R. Simms "Schoenberg, Schenker, and the Metier of Music Theory". An den Beispielen Hugo Riemanns, Arnold Schönbergs und Heinrich Schenkers diskutiert er die institutionelle Verankerung der Musiktheorie um 1900 insbesondere als Antwort auf Wilhelm Diltheys Entwurf der Geisteswissenschaften mit ihrer von Guido Adler für die Musikwissenschaft adaptierten Teilung in einen historischen und einen systematischen Zweig (die durch die neuere Musikwissenschaft in letzter Zeit immer mehr in Frage gestellt wird). Simms zeigt auf, wie Riemanns, Schönbergs und Schenkers Schriften in diese institutionellen Voraussetzungen mehr oder minder präzise eingepasst und mit Blick auf die eigene Karriere zielgerichtet für genau definierte Zwecke geschrieben sind. Schönbergs Harmonielehre von 1911 mit ihrem stufentheoretisch traditionell fundierten, dabei der Moderne zugewandten Tenor sieht Simms methodisch und inhaltlich als exakt kalkuliertes Bewerbungsschreiben bzw. als Ersatz einer Habilitationsschrift für die Wiener Akademie, wohingegen er Schenkers analytische Schriften als mit den Anforderungen der Akademie, nämlich der Möglichkeit einer Nutzung als Kompositionspropädeutika, eher inkompatibel einschätzt. Bis heute erkennt er Nachwirkungen dieser verschiedenen Ausrichtungen, die Allen Forte 
in seinem Artikel sinngemäß bestätigt (allerdings vermutet Forte dort eine etwas andere Motivation für Schönbergs Buch, das er als Antwort auf Schenkers fünf Jahre ältere Harmonielehre interpretiert, 86).

Severine Neff sucht ein oft diskutiertes gemeinsames Modell beider Theoretiker auf und führt in "Schenker, Schoenberg, and Goethe: Visions of the Organic Artwork« Schenkers und Schönbergs Zugriffe auf tonale Musik auf verschiedene Konzeptionen des Organischen bei Johann Wolfgang von Goethe zurück. Schenker wie Schönberg siedelten den Kern des Kunstwerks außerhalb der Alltagswelt an. Während Schenker jedoch den Dreiklang über dem Grundton als Archetyp setze, stelle Schönberg dem die Verhältnisse der gesamten Partialtonreihe entgegen; während Schenker im Ursatz ein universales Modell suche, sei Schönberg auf das singulare Ereignis der entwickelnd variierenden Verarbeitung eines initial vorgestellten Gedankens aus. Diese epistemologischen Fundamente verfolgt Neff in zwei Analysen von Johannes Brahms' c-MollQuartett op. 51,1, wobei sie Schönbergs Bemerkungen eine schenkerianisch gegründete Sicht in Form einer Analyse von David Gagné gegenüber stellt. Obwohl nicht direkt Schenker und Schönberg verglichen sind, ergibt sich doch eine schlüssige, methodisch tragfähige Ergänzung zu Beth Greenbergs Artikel über Schönbergs, Schenkers und Oswald Jonas' Analysen der g-Moll-Rhapsodie op. 79,2 in In Theory Only von 1975.

Martin Eybl bringt in "Schopenhauer, Freud, and the Concept of Deep Structure in Music « eine weitere Dichotomie von Welt und Gegenwelt ins Spiel. Dass sowohl Schenker als auch Schönberg die Idee einer Tiefenstruktur der Musik verfolgten, dass solche Gedanken auch Sigmund Freuds zeitgleichem Modell der menschlichen Psyche zugrunde lagen (von dem Eybl bislang unbeachtete Verbindungen zum Schönberg-Kreis ziehen kann), führt Eybl in erster Linie auf die Lektüre von Arthur Schopenhauers Die Welt als Wille und Vorstellung zurück, dies freilich unter der Prämisse einer neuen, sanalytischen` Rationalität. Begründungen für Parallelen der Ge- dankengebäude sieht er weniger in direkten Einflüssen, sondern in der gemeinsamen, dem Kommunikationsnetzwerk Wien am Beginn des 20. Jahrhunderts gewissermaßen universal eingespeisten Schopenhauer-Kenntnis (wobei im Falle Schenkers wohl auch der Einfluss von Hugo Riemanns nachhegelianischer Musikalische[r] Syntaxis von 1877 zu beleuchten wäre; dass es im 19. Jahrhundert gar keine Vorstellung von musikalischer Tiefenstruktur gegeben hätte, würde ich nicht ganz so radikal sehen wie Eybl auf S. 58).

Wie es dazu kam, dass die Wiener Universal Edition unter Emil Hertzka zum gemeinsamen Verlag der Antipoden Schenker und Schönberg wurde, untersucht Christopher Hailey (»Anbruch and Tonwille: The Verlagspolitik of Universal Edition «), und schon die dort zitierte, herrlich treffende Schenker-Parodie aus der Karnevalsausgabe 1925 der Musikblätter des Anbruch (in der speziellen Ausgabe: Abbruch, 64) lohnt die Lektüre. Aber Hailey zeigt vor allem auf, wie die Universal Edition eine ästhetisch widersprüchliche Verlagspolitik»[b] etween popular and rigorous scholarly editions, between hallowed classics and radical modernists, between Anbruch and Tonwille« betrieb (wobei die erstgenannten Punkte als Fortsetzung der Mischkalkulation nichts Einzigartiges waren; das Nebeneinander sich widersprechender musiktheoretischer Ansätze im gleichen Verlag kennt man von der Veröffentlichung der Schriften Moritz Hauptmanns und Simon Sechters 1853/54 bei Breitkopf \& Härtel in Leipzig) sthat went far beyond the cultivation of one city's claims to a musical past to demonstrate how creative thinking about that past might stimulate its legacy to the future $(67)$.

Unter der Leitfrage »Atonal Prolongation - eine Chimäre?« stellt Gerold W. Gruber Diskussionsbeiträge von Autoren der SalzerNachfolge und ihren Opponenten zu dem Problem zusammen, ob eine modifizierte Schenker-Theorie insbesondere in der Musik Schönbergs Klangprolongationen erfassen könne. Auf diese Frage (und die Diskussionen um Felix Salzer und Roy Travis) kommen auch andere Autoren des Bandes mehrmals zu 
sprechen. Neben Salzer und Travis nennt Gruber Allen Forte als einflussreichen Vertreter dieses Ansatzes. Dessen eigene, oben schon kurz gestreifte Darstellung "Schenkerians and Schoenbergians in America" schließt die Schönberg-Sektion des Buchs ab und leitet bereits zur zweiten über, wo Forte aus eigener Erinnerung über das Wirken von Protagonisten beider Denkrichtungen (und über die Mödlinger Müllabfuhr) berichtet. Seine Ausführungen erhellen die Trennung beider Schulen und ihre inhaltliche Affinität zu verschiedenen Subdisziplinen (die der Schenkerianer zu Analyse und Aufführung, die der Schönbergianer zur Komposition), aber auch seine eigenen, von ihm nur sehr bescheiden angedeuteten Ansätze zur Versöhnung der Gegensätze.

\section{II. »Proponenten und Schulen«}

Die sieben Studien zu »Proponenten und Schulen« (deren mitunter anekdotischer Tonfall in einer musiktheoretischen Publikation zunächst verwundern mag, aber durch die umso direktere Darstellungsweise auch zum Reiz der Darstellungen beiträgt) beginnen mit David Carson Berrys Gedanken zu Hans Weisse, der bereits im Herbst 1931, also noch zu Schenkers Lebzeiten, an der David Mannes Music School, ab 1932 schließlich an der Columbia University unterrichtete und dadurch den Anfang der US-amerikanischen SchenkerRezeption der 1930er-Jahre bestimmte. Sein Einfluss auf die Publikation der Fünf UrlinieTafeln wird im gleichen Band auch von Hedi Siegel hervorgehoben. Obwohl im Rückblick Weisse von seinem ehemaligen Schüler und späteren Nachfolger Salzer weitgehend überschattet wird, ist doch in der Liste seiner Schülerinnen und Schüler (darunter neben Salzer Adele Katz, Autorin des ersten englischsprachigen Schenker-Lehrbuchs Challenge to $\mathrm{Mu}$ sical Tradition. A New Concept of Tonality, das Oswald Jonas scharf ablehnte) der Einfluss seines Unterrichts deutlich zu greifen.

Felix Salzers eminent folgenreiches pädagogisches Wirken in New York untersucht kein Geringerer als Carl Schachter. Salzer konzipierte an der Mannes School einen musiktheoretischen Lehrgang nach Schenker, aus dem 1952 schließlich das im Einfluss kaum zu überschätzende Buch Structural Hearing erwuchs. Trotz scharfen Gegenwinds (insbesondere von Oswald Jonas und Ernst Oster, die Salzers gravierende Änderungen an Schenkers Theorie nicht hinnehmen wollten) bereitete diese Publikation den Boden für die intensive amerikanische Schenker-Rezeption ab den 1970er-Jahren.

John Rothgeb rekapituliert das Schaffen von Oswald Jonas, der mit Das Wesen des musikalischen Kunstwerks bereits 1934 eine Einführung in das Denken Schenkers vorlegte. Jonas' Rolle bei der Editionsgeschichte der Übersetzung von Schenkers Harmonielehre wird in der dritten Sektion des Bandes von Robert W. Wason untersucht. Vehement hebt Rothgeb zunächst auf Jonas' ablehnende Haltung gegenüber Schenkers politischer Ideologie ab, schön zusammengefasst in einer Glosse seines Der Tonwille-Handexemplars zu Schenkers Artikel "Von der Sendung des deutschen Genies«: "heilloser Unsinn«, schrieb Jonas dazu kurz, bündig und treffend. Schwerpunkte seiner Arbeit waren die Verbindung von Analyse und Aufführung sowie die Liedanalyse, wobei Rothgeb Jonas' Unterrichtsweise, in der das Klavier eine größere Rolle spielte als Stimmführungsdiagramme, als Charakteristikum hervorhebt.

Letzteres unterscheidet Jonas von seinem Schüler Ernst Oster, über dessen Tätigkeit William Rothstein berichtet. Oster sieht er als Leitfigur der Weitergabe von Schenkers Lehre im originalen Zuschnitt (in technischer, nicht in kontextueller Hinsicht, wie Robert P. Morgan später ergänzt - da habe Oster, ähnlich wie Jonas, eine rigorose ideologische Zensur vorgeschaltet): Nicht nur bewahrte er den größten Teil von Schenkers Nachlass (die Ernst Oster Collection ist neben den Sammlungen von Oswald Jonas und Felix Salzer heute einer der Grundpfeiler der historischen SchenkerForschung), er übersetzte auch Der freie Satz ins Englische und beteiligte sich maßgeblich an der Diskussion um Salzers und Travis' Ausweitung der Schenker-Analyse auf Musik des 
frühen 20. Jahrhunderts. Versuchen einer Popularisierung und Vereinfachung der Theorie stand Oster, der Analysen schwieriger Passagen jahrzehntelang immer wieder überdenken und verfeinern konnte, abwehrend gegenüber. Als unvermutete, aber faszinierende Zugabe teilt Rothstein im Anhang Osters Analyse der Takte 35 bis 42 aus Ludwig van Beethovens Klaviersonate C-Dur op. 53 (der ,Waldstein «Sonate) mit: ein spannender Einblick in Osters analytische Werkstatt und sein kompromissloses Streben nach einer möglichst perfekten, dabei erstaunlich komplexen Erklärung dieser wenigen (aber problematischen) Takte.

Wolfgang Suppans engagiert geschriebenes Porträt von Viktor Zuckerkandl geht über die Themenstellung hinaus und nimmt auch die skeptische Schenker-Sicht großer Teile der deutschsprachigen Musikwissenschaft nach dem Krieg kurz in den Blick. Zudem ermöglicht es einen exemplarischen Einblick in Schenkers Wirkung über den engeren Kreis der Musiktheorie hinaus auf Philosophie und Ästhetik; schade, dass nicht auch Gerhard Albersheims grundsätzlich ähnliche Ansätze einer Übertragung auf die Musikpsychologie vorgestellt werden.

Es folgen zwei Überblicksdarstellungen. Zunächst skizziert Patrick Boenke in seinem Beitrag "Zur österreichischen und deutschen Rezeption der Schichtenlehre Heinrich Schenkers « Stationen der deutschsprachigen Schenkertradition. Boenke verweist auf Moriz Violin und Felix-Eberhard von Cube von 1935 bis 1938 und ab 1947 in Hamburg, auf Franz Eibner im Wien der 1950er-Jahre und auf Hellmut Federhofer in Graz und Mainz. Leider werden diese Sachverhalte alle nur beiläufig gestreift. Es fehlt der Name Reinhard Oppels, der ab 1927 am Konservatorium Leipzig lehrte. Auch Wilhelm Furtwänglers Eintreten für Schenker wäre erwähnenswert gewesen, und die Begründungen der weithin negativen deutschsprachigen SchenkerRezeption der Nachkriegszeit scheinen mir, so weit sie über dumpf weiter schwelenden Antisemitismus hinausgingen, gleichfalls einer Untersuchung wert: etwa in Form einer Aufbereitung der Schenker-Skepsis von Theodor
W. Adorno und Carl Dahlhaus, wie sie sich in der Kontroverse von Dahlhaus mit Federhofer und Karl-Otto Plum darstellte. Evelyn FinkMennel schreibt kundig über »Das SchenkerInstitut und der Lehrgang für Tonsatz nach Heinrich Schenker. Zur Wiener SchenkerTradition" und geht auch auf das 1935 gegründete Schenker-Institut am Neuen Wiener Konservatorium ein. Insbesondere widmet sie sich Franz Eibner, der nach dem Krieg Schenkers Theorie in Wien institutionell verankern konnte. Eine erweiterte Fassung ihres Textes war schon 2003 im eingangs erwähnten Ausstellungskatalog erschienen.

\section{III. "Schenker-Lektüre in den USA: Editionen und Übersetzungen«}

Die letzten Artikel des Bandes sind als "Schenker-Lektüre in den USA: Editionen und Übersetzungen« rubriziert. Robert W. Wason dokumentiert in »From Harmonielehre to Harmony: Schenker's Theory of Harmony and Its Americanization « nicht nur einen frühen Entwurf Schenkers zu diesem Buch, sondern auch die Veröffentlichungs- und Übersetzungs- (und damit eine Voraussetzung für die Rezeptions-)Geschichte. Wason gelingt eine fulminante Studie zu Genese und Wirkung des Textes, die wichtige handschriftliche Quellen (Schenkers durchschossenes Handexemplar und Otto Vrieslanders Ergänzungen) zu einem faszinierenden Bild zusammenfügt.

Hedi Siegel kehrt nochmals zurück zum Beginn der amerikanischen Wirkungsgeschichte. "The Pictures and Words of an Artist ('von einem Künstlers): Heinrich Schenker's Fünf Urlinie-Tafeln« erkennt sie als Kristallisationspunkt der frühen Schenker-Rezeption. Das den Urlinie-Tafeln beigefügte Glossar habe die Formierung der englischsprachigen Analyseterminologie bis heute geprägt. Aber vor allem hebt sie hervor, dass gerade diese Veröffentlichung (gemeinsam mit dem Beispielband zu Der freie Satz) eine sgrafische، Wirkungsweise erlaubte, die die erste Fühlungnahme mit Schenkers Theorie von Übersetzungen und deutschen Sprachkenntnissen unabhän- 
gig machte: »The spictures` came first, and the words came later« (218).

Zuletzt untersucht Robert P. Morgan "Schenker's Der freie Satz: History, Significance, Reception «. Auch wenn er den Einfluss von Osters posthum erschienener Übersetzung auf die amerikanische Musiktheorie der 1980er-Jahre eingangs etwas relativiert, sieht er das Buch als Grundlage eines zeitgemäßen Verständnisses der Schichtenlehre und plädiert dafür, auch die ideologisch belasteten Partien historisch offensiv als "part and parcel of the theory's gestation« (230) zu verstehen (und dann im Wissen darum gegebenenfalls zu verwerfen), statt sie im Vorhinein in Neuausgaben stillschweigend $\mathrm{zu}$ unterdrücken: Osters analysetechnische Brillanz sei mit einer in bester Absicht vorgenommenen verfälschenden Bereinigung der geistesgeschichtlichen Kontexte einhergegangen.

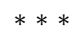

Eine Zusammenstellung der Lebensläufe von 15 Schülerinnen und Schülern Schenkers, ein Verzeichnis der Autorinnen und Autoren des Bandes sowie ein Namens- und Ortsregister runden das Buch ab. Die CD-ROM bringt eine Fülle von Informationen aus der Wiener Ausstellung, sogar Schenkers eigene Klavierstücke op. 1 komplett mit Noten und Toneinspielung, und provoziert geradezu, dass man längere Zeit in ihr versinkt (allerdings auch, weil die Menüführung anfangs ein bisschen verwir- rend ist; Linux-Nutzer sind leider außen vor gelassen).

Insgesamt ist Eybl und Fink-Mennel ein so informatives wie kurzweiliges Buch gelungen. Dass nicht alle Wünsche erfüllt werden können, liegt bei einer Aufsatzsammlung in der Natur der Sache - es hätten sicher noch weitere für die Wirkungsgeschichte Schenkers relevante Personen gesondert vorgestellt werden können (Albersheim, von Cube, Federhofer, Oppel, Roth), die ohnehin spärliche Rezeption im deutschsprachigen Raum erscheint mir dadurch etwas unterbelichtet und der Grundton des Buchs gerät bisweilen allzu apologetisch (auch negative Rezeption ist Rezeption). Mittlerweile bieten sich die rezeptionsgeschichtlichen Artikel der ersten zwei Bände der Zeitschrift der Gesellschaft für Musiktheorie sowie Nicholas Cooks jüngst erschienenes Buch The Schenker Project als vertiefende Lektüre zur rezeptionsgeschichtlichen Kontextualisierung und geistesgeschichtlichen Verortung ebenso an wie Ian Bents Schenker Correspondence Project im Internet in letzter Zeit neue Quellen bereitgestellt hat. Nichts desto trotz handelt es sich um einen lehrreichen und schön ausgestatteten Sammelband, der als exemplarische Wissenschafts- und Rezeptionsgeschichte mit Schwerpunkt Exilforschung sowie als Zeichen der inhaltlichen Bandbreite und Vitalität von Ansätzen zur Schichtenanalyse unbedingt (beileibe nicht nur für Musikwissenschaftlerinnen und Musikwissenschaftler mit musiktheoretischem Schwerpunkt) lesenswert ist.

Christoph Hust 